\title{
Eine Berechnung der Feldgradienten am Ort der Fe- und Co-Kerne in $\mathrm{Fe}\left(\mathrm{C}_{5} \mathrm{H}_{5}\right)_{2}$ und $\left[\mathrm{Co}\left(\mathrm{C}_{5} \mathrm{H}_{5}\right)_{2}\right]^{+}$
}

\author{
III. Sternheimer-Korrektur der Feldgradienten \\ Von Bernd Höfrlinger * und Jürgen Voitländer \\ Aus dem Physikalisch-Chemischen Institut der Universität München \\ (Z. Naturforschg. 18 a, 1185-1190 [1963] ; eingegangen am 17. September 1963)
}

\begin{abstract}
An estimate of Sternheimer polarisation effects in the electronic core of the central atoms is given in this concluding part of our series on the field gradients in $\mathrm{Fe}\left(\mathrm{C}_{5} \mathrm{H}_{5}\right)_{2}$ and $\left[\mathrm{Co}\left(\mathrm{C}_{5} \mathrm{H}_{5}\right)_{2}\right]^{+}$. The Sternheimer factors used for inter- and extrapolations are those obtained in the literature by the perturbation-variation method without exchange. The results are mainly of a qualitative importance: The field gradient including Sternheimer correction is in $\left[\mathrm{Co}\left(\mathrm{C}_{5} \mathrm{H}_{5}\right)_{2}\right]^{+}$e $q=1.67 \mathrm{a}$. $\mathrm{u}$. experimentally and $e q=1.06 \mathrm{a}$. u. theoretically. The theoretical results for $\mathrm{Fe}\left(\mathrm{C}_{5} \mathrm{H}_{5}\right)_{2}$ are less reliable because of an obvious uncertainty in the choice of LCAO-coefficients and a merely approximate computation of the two-center terms in the expansion of the field gradient: $e q=0.33 \mathrm{a}$. u. theoretically and $e q=1.25$ a. u. experimentally for a $\mathrm{Fe}^{57 \mathrm{~m}}$ quadrupole moment of $0.18 \mathrm{~b}$. A summation of important facts in all three parts of this investigation is given as conclusion.
\end{abstract}

Im ungestörten Zustand besitzen abgeschlossene Elektronenschalen eines Atoms Kugelsymmetrie und treten daher nicht in orientierungsabhängige, also z. B. Quadrupolwechselwirkungen mit benachbarten Ladungsverteilungen, z. B. einem Atomkern mit elektrischem Quadrupolmoment oder einem nicht kugelsymmetrischen System von umgebenden Elektronen und Ionen. Die Energie des Gesamtsystems verringert sich dadurch, daß die unsymmetrischen Ladungsverteilungen die Kugelsymmetrie der abgeschlossenen Schalen, des Elektronenrumpfs, zerstören. Der gestörte Rumpf nimmt an Quadrupolwechselwirkungen teil. Der rotationssymmetrische Teil des Quadrupolwechselwirkungsoperators ist in Polarkoordinaten $H_{Q} \sim\left(3 \cos ^{2} \Theta_{\mathrm{c}}-1\right)\left(3 \cos ^{2} \Theta-1\right)\left\{\begin{array}{ll}r_{\mathrm{c}}{ }^{2} / r^{3}, & r_{\mathrm{c}}<r \\ r^{2} / r_{\mathrm{c}}^{3}, & r<r_{\mathrm{c}}\end{array}\right.$.

Durch die Koordinaten $r_{\mathrm{e}}, \Theta_{\mathrm{e}}$ ist der auf ein Rumpfelektron wirkende Teil bezeichnet. Nach Bildung der Erwartungswerte zeigt sich, daß eine die Gesamtenergie verringernde Störungsenergie erreicht wird

1. im Angularteil durch eine $l^{\prime}$-Störung der $n l$ Rumpfschalen mit $l^{\prime}=l \pm 2$, die man als angulare Anregung bezeichnet und mit $\left(n l \rightarrow l^{\prime}\right)$ symbolisiert,

2. im Radialteil durch Störung der Radialfunktionen einer Rumpfschale, bezeichnet als radiale Anregung und symbolisiert durch $(n l \rightarrow l)$.

Angulare Anregungen erweisen sich immer als „shielding“, d. h. der Feldgradient einer äußeren

* Teil einer Diplomarbeit am Physikalisch-Chemischen Institut der Universität München.
Ladungsverteilung erscheint am Kernort verkleinert. Radiale Anregungen bedeuten „antishielding“ für die äußere Ladungsverteilung, wenn diese im wesentlichen außerhalb des Rumpfes liegt, und leichtes "shielding“, wenn sie stark genug in den Rumpf eindringt.

Die Störungsrechnung erbringt für den gestörten Rumpf einen induzierten Feldgradienten $q_{\mathrm{c}}$, der proportional zum Feldgradienten $q_{\mathrm{i}}$ der störenden Verteilung $\mathrm{i}$ ist:

$$
q_{\mathrm{c}}=\gamma q_{\mathrm{i}} ; \quad \gamma \equiv \sum_{n l \rightarrow l^{\prime}} \gamma\left(n l \rightarrow l^{\prime}\right), \quad l^{\prime}=l, l \pm 2 .
$$

Der Sternheimer-Faktor $\gamma$ ist eine rein radial abhängige Funktion, $d$. h. für eine äußere Punktladung in $\left(r_{\mathrm{i}}, \Theta_{\mathrm{i}}, \Phi_{\mathrm{i}}\right)$ ist $\gamma=\gamma\left(r_{\mathrm{i}}\right)$.

$\mathrm{Zu}$ dem Quadrupolmoment, das vom Kernquadrupolmoment $Q$ im Rumpf induziert wird, stellt die Störungsrechnung fest, daß das induzierte Quadrupolmoment $\gamma Q$ mit dem äußeren Feldgradienten $q_{\mathrm{i}}$ die gleiche Wechselwirkung produziert, wie der von außen im Rumpf induzierte Feldgradient $\gamma q_{\mathrm{i}}$ mit dem Kernquadrupolmoment. Zur Ermittlung von $\gamma$ wird hier der zweite Standpunkt gewählt.

Treten Übergangsmetallatome als Zentralatome in kovalenten Molekülen auf, so ist deren Elektronenrumpf mit Einschluß der 3p-Elektronen ein ${ }^{1} \mathrm{~S}$ Rumpf, auf den Quadrupolstörungen wirken. Von bisherigen Ergebnissen für die Sternheimer-Faktoren sind diejenigen am Beginn der Übergangsreihe, wo die Elektronenkonfiguration ...3 $3 \mathrm{p}^{6} 3 \mathrm{~d}^{0}$ ist, direkt verwendbar. Ergebnisse für $\ldots 3 \mathrm{p}^{6} 3 \mathrm{~d}^{5}$. und 
$\ldots 3 \mathrm{p}^{6} 3 \mathrm{~d}^{10}$-Konfigurationen müssen insofern reduziert werden, als in ihnen zunächst die Quadrupolstörung der $3 \mathrm{~d}^{5}$-Unterschale bzw. der ganzen $3 \mathrm{~d}^{10}$. Schale mit eingeschlossen ist. Diese Reduktion ist dann leicht möglich, wenn für jede Schale des Rumpfes der Polarisationsbeitrag explizit bekannt ist, wie das z. B. für radiale Anregungen meistens der Fall ist, und wenn ein Störungsmodell verwendet wurde, das Rückwirkungen von $3 \mathrm{~d}^{5}$ bzw. $3 \mathrm{~d}^{10}$ auf innere Schalen nicht berücksichtigt, das also keinen SCF. Formalismus enthält. Damit ist man auf das Niveau der Störungsrechnungen ohne Rückwirkungen und Austausch verwiesen. Wenn man noch darauf achtet, daß jeweils die Anwendung des gleichen Verfahrens die Ergebnisse für verschiedene Atome und Ionen vergleichbar macht, so ergibt sich das umfangreichste Wertesystem, wenn die $\gamma_{\infty}$-Werte von WIKNER und $\mathrm{D}_{\text {AS }}{ }^{1}$ zugrunde gelegt werden, die K II, Cu II und Ga IV betreffen. Sie werden ergänzt durch die $\gamma_{\infty}$. Werte für Mn III und Fe IV von Burns und WIKNER ${ }^{2}$, denen ebenfalls der Variationsansatz von DAS und BERsoHN ${ }^{3}$ und Ingalls' ${ }^{4}$ Orthogonalisierungsmethode a) zugrunde liegt. In diesen Rahmen fügt sich dann auch der Fe III-Wert von Ingalls ${ }^{4}$ nach Methode a) ein:

$\begin{array}{ccccccc} & \text { K II } & \text { Mn III } & \text { Fe III } & \text { Fe IV } & \text { Cu II } & \text { Ga IV } \\ \gamma_{\infty}(\mathrm{rad}) & 14,25 & 5,50 & 6,71 & 5,68 & 6,37 & 4,82\end{array}$

Bedenken gegen dieses System von $\gamma_{\infty}$-Werten werden durch folgende Vergleiche nahegelegt:

1. Die $\gamma_{\infty}$-Werte von Sternheimer ${ }^{5,6}$ fallen prinzipiell größer aus als die zitierten Variationsergebnisse und erscheinen insofern verläßlicher, als sie die exakte Lösung der Störungsrechnung darstellen und von $\mathrm{W}_{\text {atson }}$ und Freeman ${ }^{7}$ bestätigt werden.

2. Die Ergebnisse von $W_{\text {Atson }}$ und $F_{\text {Reeman }} 7,8$ weisen darauf hin, daß im Variationsverfahren der Störungsrechnung die simultane Orthogonalisierung, Ingalls' ${ }^{4}$ Methode b), die richtige ist. Diese liefert höhere $\gamma_{\infty}$-Werte als die Methode a) der sukzessiven Orthogonalisierung. Übereinstimmung mit den Ergebnissen von Sternheimer würde aber auch dann noch nicht erreicht.

1 E. G. Wikner u. T. P. Das, Phys. Rev. 109, 360 [1957].

2 G. Burns u. E. G. Wikner, Phys. Rev. 121, 155 [1961].

3 T. P. Das u. R. Bersohn, Phys. Rev. 102, 733 [1956].

4 R. Ingalls, Phys. Rev. 128, 1155 [1962].

5 H. M. Foley, R. M. Sternheimer u. D. Tycko, Phys. Rev. 93, 734 [1954].
Angeleitet von der Tatsache, daß die $\gamma_{\infty}$-Werte sich in Abhängigkeit von Ordnungs- und Elektronenzahl der Atome oder Ionen um größenordnungsmäßig eine Zehnerpotenz ändern, werden die $\gamma_{\infty}(\mathrm{rad})$ Ergebnisse in der Übergangsmetallreihe halblogarithmisch über der Ordnungszahl aufgetragen und Punkte gleicher Ionisationsstufe geradlinig verbunden (Abb. 1). Der mit Hilfe des Thomas-Fermi-Modells berechenbare ${ }^{9} \gamma_{\infty}$ (ang)-Wert $-1,04^{2}$ für Fe IV $3 d^{5}$

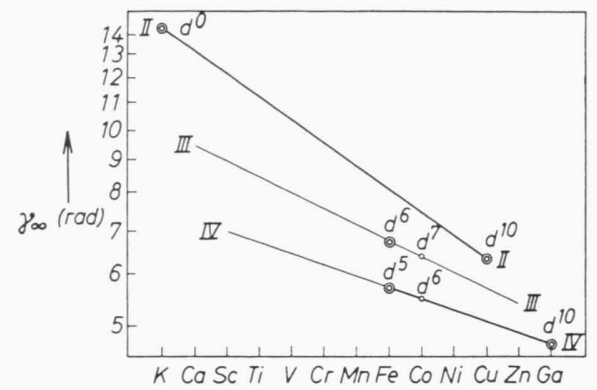

Abb. 1. Antishielding Faktoren der ... 3 p $^{6}$-Rümpfe von Übergangsmetallionen.

soll für Fe III $3 \mathrm{~d}^{6}$ als Faktor der angularen Anregungen, einschließlich der 3p-Elektronen, gelten. Zunahme des Wertes als Folge der um eins niedrigeren Ionisationsstufe und Abnahme des Wertes wegen des Ausschlusses der $3 \mathrm{~d}^{5}$-Unterschale sollen sich also gerade aufheben. Zieht man noch $\gamma_{\infty}($ ang $)=-1,08^{2}$ für Mn III $3 \mathrm{~d}^{5}$ heran und extrapoliert linear, so ist z. B. für Co IV $\gamma_{\infty}$ (ang) $=-1,0$ für den Rumpf mit Einschluß der 3p-Elektronen zu wählen.

Informationen über den schematischen Verlauf von $\gamma(r)$ erhält man aus der Darstellung für Cu II von Foley, Sternheimer und Tycko ${ }^{5}$. Von Interesse ist die Stelle des shielding-Maximums, die für Übergangsmetalle bei $r \sim 0,5$ at. E. liegt, und die Nullstelle bei $0,8-1,0$ at. E. Diese wird im folgenden bei 1,0 at. E. angenommen, da z. B. die Halbwertshöhe von $P_{3 \mathrm{p}}^{2}(r)=r^{2} R_{3 \mathrm{p}}^{2}(r)$ für Fe III bei $r=1,08$ at. E. liegt, dort also sicher schon das antishielding begonnen hat. Es wird angenommen, daß

$$
\gamma_{3 \mathrm{~d}} \equiv \frac{\left\langle\gamma(r) r^{-3}\right\rangle_{3 \mathrm{~d}}}{\left\langle r^{-3}\right\rangle_{3 \mathrm{~d}}}
$$

${ }^{6}$ R. M. Sternheimer u. H. M. Foley, Phys. Rev. 102, 731 [1956]. - R. M.Sternheimer, Phys. Rev. 115, 1198 [1959]; 130, 1423 [1963].

7 R. E. Watson u. A. J. Freeman, Teil I einer demnächst in Phys. Rev. erscheinenden Arbeit.

8 A. J. Freeman u. R. E. Watson, Teil II einer demnächst in Phys. Rev. erscheinenden Arbeit.

9 R. M. Sternheimer, Phys. Rev. 80, 102 [1950]. 
jeweils das shielding-Maximum darstellt. Darauf weist die Näherung $\gamma_{3 \mathrm{~d}} \sim \gamma\left(r_{3 \mathrm{~d}}^{\max }\right)$ hin, die wegen $\left\langle r^{-3}\right\rangle_{3 \mathrm{~d}} \sim\left(r_{3 \mathrm{~d}}^{\max }\right)^{-3}$ annehmbar erfüllt ist $\left(r_{3 \mathrm{~d}}^{\max }\right.$ ist die Stelle des Maximums von $P_{3 \mathrm{~d}}(r)$ und liegt für Fe III und Co IV bei $\sim 0,6$ at. E.). Die Ergebnisse von Freeman und Watson ${ }^{8}$ legen hier die Wahl der INGalls-Ergebnisse ${ }^{4}$ für Fe III bei simultaner Orthogonalisierung nahe, die wieder um den $3 \mathrm{~d}^{5}$. Beitrag zu reduzieren sind. Schließlich bleibt noch festzustellen, daß $\left[P_{3 \mathrm{p}}(r)\right]^{2}$ bei $r \sim 3$ at. E. effektiv Null wird, so daß dort $\gamma_{\infty}$ erreicht ist. Diese Schätzungen werden zur Herstellung von Abb. 2 benutzt.

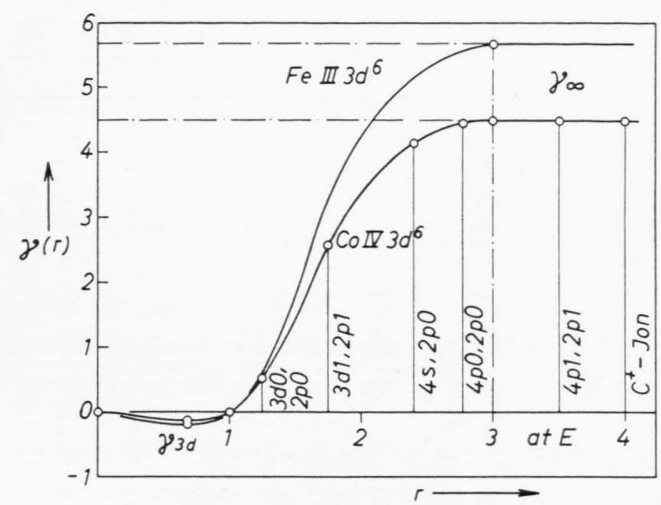

Abb. 2. Die Polarisationsfunktion $\gamma(r)$ für Fe III und Co IV.

Der Beitrag der Rumpfpolarisation zum Feldgradienten am Kernort der Fe- bzw. Co-Atome in den betrachteten Sandwich-Molekülen wird jetzt im Anschluß an Gl. (1) von Teil II der hier wiedergegebenen Arbeit ${ }^{10}$ durch folgenden modellmäßigen Ansatz bestimmt:

$$
\begin{aligned}
e q_{\mathrm{c}}= & -e \sum_{j} N(j) \\
& \frac{\int \psi_{j}^{*} \frac{\gamma(r)}{r^{3}} \psi_{j} \mathrm{~d}^{3} \boldsymbol{r}}{\int \psi_{j}^{*} \frac{1}{r^{3}} \psi_{j} \mathrm{~d}^{3} \boldsymbol{r}} \int \psi_{j}{ }^{*} \frac{3 \cos ^{2} \Theta-1}{r^{3}} \psi_{j} \mathrm{~d}^{3} \boldsymbol{r} \\
& +10 e \gamma_{\infty} \frac{3 \cos ^{2} \omega-1}{\varrho^{3}} .
\end{aligned}
$$

Im Hinblick auf den ersten Summanden, der in Valenzelektronen-, Überlappungs- und Ligandenelektronenbeitrag zerfällt (Abschnitt VI von Teil II), sind also erneut radiale Integrationen auszuführen. Die Radialfunktionen der 3d- und weiter innen liegenden Zentralatomelektronen werden von der jeweili-

10 B. Höfflinger u. J. Voitländer, Z. Naturforschg. 18 a, 1065 [1963], bezeichnet als Teil I; 18 a, 1074 [1963], bezeichnet als Teil II. gen $\ldots 3 \mathrm{p}^{6} 3 \mathrm{~d}^{\mathrm{x}}$-Konfiguration bestimmt, die $4 \mathrm{~s}$ - und $4 p$-Elektronenbesetzung hat also vernachlässigbaren Einfluß (Abschnitt VII von Teil I). Da die Populationsanalyse (Abschnitt II von Teil II) 6,05 als Besetzungszahl der $3 \mathrm{~d}$-Schale ergeben hat, können direkt die $\gamma(r)$-Darstellungen der Abb. 2 benutzt werden. Mit deren shielding-Maxima ist das Problem der 3d-Valenzelektronen bereits gelöst. Die Hauptmaximumsnäherung

$$
\gamma_{n l} \approx \frac{\gamma\left(r_{n l}^{\max }\right)}{\left(r_{n l}^{\max }\right)^{3}} \frac{1}{\left\langle r^{-3}\right\rangle_{n l}}
$$

$\left[r_{n l}^{\max }\right.$ bezeichnet die Lage des Hauptmaximums von $\left.P_{n l}(r)\right]$ von Sternheimer ${ }^{11}$ ergibt $\mathrm{z}$. B. für die von der Konfiguration $\mathrm{Co}_{\mathrm{B}}$ (Abschnitt II von Teil II) bestimmte $4 \mathrm{p}$-Radialfunktion $r_{4 \mathrm{p}}^{\max }=2,71$ at. E. (Abb. 4 von Teil II), $\gamma(2,71)=4,4$ (Abb. 2), $\left\langle r^{-3}\right\rangle_{4 \mathrm{p}}=3,54$ at. E. (Abschnitt VI von Teil II)

$$
\gamma_{4 \mathrm{p}} \approx \frac{4,4}{(2,71)^{3} \cdot 3,54}=0,062
$$

und entsprechend für den $\mathrm{Fe}_{\mathrm{B}}$-Fall: $\gamma_{4 \mathrm{p}}=0,142$. Eine genauere Bestimmung von $\gamma_{n l}$ kann durchgeführt werden, wenn $P_{n l}(r)=r R_{n l}(r)$ durch

$$
R_{n l}(r)=\sum_{k} c_{k} \chi_{k}(r)
$$

dargestellt ist, worin $\chi_{k}$ normierte STO's sind. Denn wegen [vgl. Gl. (2) von Teil I]

$$
\left\langle r^{-3}\right\rangle_{n l}=\int_{0}^{\infty} P_{n l}^{2}(r) r^{-3} \mathrm{~d} r \equiv \sum_{i, k} q^{i, k}
$$

kann man schreiben

$$
\frac{\left\langle\gamma(r) r^{-3}\right\rangle_{n l}}{\left\langle r^{-3}\right\rangle_{n l}} \approx \sum_{i, k} \gamma\left(r_{i, k}^{\max }\right) q^{i, k},
$$

worin $r_{i, k}^{\max }$ das Maximum von $r^{2} \chi_{i}(r) \chi_{k}(r)$ bezeichnet. Nach dieser Methode erhält man z. B. für $\mathrm{Co}_{\mathrm{B}}$ $\gamma_{4 \mathrm{p}}=0,078$, also größenordnungsmäßige Übereinstimmung mit dem obigen Ergebnis der Hauptmaximumsnäherung. Dies legt sogleich den Versuch nahe, die Quadrupolstörungsfaktoren der Überlappungs- und Ligandenelektronen-Verteilungen aus deren Punktladungsnäherungen (Anhang $\mathrm{C}$ von Teil II) zu bestimmen. Wenn die betrachtete Überlappungsverteilung in radialer Richtung effektiv nur wenig ausgedehnt ist, wie das z. B. bei $\pi$-Überlappungen der Fall ist, oder wenn sie hauptsächlich im

11 R. M. Sternheimer, Phys. Rev. 105, 158 [1957]. 
Proportionalitätsbereich von $\gamma(r)$ liegt, ist das Verfahren vertretbar. Auch die Mulliken-Näherung (Anhang D von Teil II) könnte verwendet werden. Sie würde hier lauten:

$$
\begin{aligned}
& \gamma_{n l m, n^{\prime} l^{\prime} m^{\prime}} q^{n l m, n^{\prime} l^{\prime} m^{\prime}} \approx \frac{1}{2}\left\langle n l m \mid n^{\prime} l^{\prime} m^{\prime}\right\rangle \\
&\left(\gamma_{n l m} q^{n l m}+\gamma_{n^{\prime} l^{\prime} m^{\prime}, n^{\prime} l^{\prime} m^{\prime}} q^{n^{\prime} l^{\prime} m^{\prime}, n^{\prime} l^{\prime} m^{\prime}}\right) .
\end{aligned}
$$

Sechsfach indizierte Größen sind Zweizentrenterme. Die Ergebnisse zeigen geringe Übereinstimmung mit denen der wesentlich verläßlicheren „Näherung durch Separation" (Anhang E von Teil II). Da die dortigen radialen Integrationen neben der analytischen Berechnung auch numerisch ausgeführt wurden, können die jeweiligen $\gamma(r)$-Faktoren nachträglich leicht ergänzt und damit Sternheimer-Faktoren für die Beiträge $q^{3 \mathrm{~d} 0,2 \mathrm{p} 0}, q^{4 \mathrm{~s}, 2 \mathrm{p} 0}, q^{4 \mathrm{p} 0,2 \mathrm{p} 0}$ und $q^{2 \mathrm{p} 0,2 \mathrm{p} 0}$ erhalten werden. Nach Transformation auf die Hauptachse der Moleküle ergeben sich dann die STERNHeIMER-Faktoren der Tab. 1 und 2, die zugleich korrigierte resultierende Feldgradienten für die Moleküle $\left(\mathrm{CoCp}_{2}\right)^{+}$und $\mathrm{FeCp}_{2}$ angeben.

\begin{tabular}{|l|c|c|c|}
\hline & $e q$ & $\gamma$ & $(1+\gamma) e q$ \\
\hline $3 \mathrm{~d}$ & $+2,979$ & $-0,153$ & $+2,523$ \\
$4 \mathrm{p}$ & $+0,175$ & $+0,078$ & $+0,189$ \\
$\mathrm{C}^{+}-$Ionen & $+0,159$ & $+4,5$ & $+0,875$ \\
$2 \mathrm{p}, 2 \mathrm{p}$ & $-0,274$ & $+4,25$ & $-1,439$ \\
$3 \mathrm{~d} \pm 1, \mathrm{e}_{1 \mathrm{~g}}$ & $-0,384$ & $+0,021$ & $-0,392$ \\
$3 \mathrm{~d} \pm 2, \mathrm{e}_{2 \mathrm{~g}}$ & $-0,176$ & $+0,084$ & $-0,191$ \\
$4 \mathrm{~s}, \mathrm{a}_{1 \mathrm{~g}}$ & $-0,025$ & $+3,00$ & $-0,100$ \\
$4 \mathrm{p} 0, \mathrm{a}_{2 \mathrm{u}}$ & $-0,037$ & $+3,63$ & $-0,171$ \\
$4 \mathrm{p} \pm 1, \mathrm{e}_{1 \mathrm{u}}$ & $-0,050$ & $+3,77$ & $-0,238$ \\
\cline { 2 - 3 } gesamt & $+2,367$ & & $+1,056$ \\
& at. E. & & at. E. \\
\hline
\end{tabular}

Tab. 1. Sternheimer-Korrektur der Feldgradientenbeiträge in $\left(\mathrm{CoCp}_{2}\right)^{+}$.

\begin{tabular}{|l|c|c|c|}
\hline & $e q$ & $\gamma$ & $(1+\gamma) e q$ \\
\hline $3 \mathrm{~d}$ & $+2,340$ & $-0,186$ & $+1,905$ \\
$4 \mathrm{p}$ & $+0,095$ & $+0,141$ & $+0,108$ \\
$\mathrm{C}^{+}-$Ionen & $+\mathbf{0 , 1 7 0}$ & $+5,67$ & $+1,133$ \\
$2 \mathrm{p}, 2 \mathrm{p}$ & $-0,27$ & $+5,25$ & $-1,69$ \\
$3 \mathrm{~d} \pm 1, \mathrm{e}_{1 \mathrm{~g}}$ & $-0,46$ & $+0,02$ & $-0,47$ \\
$3 \mathrm{~d} \pm 2, \mathrm{e}_{2 \mathrm{~g}}$ & $-0,21$ & $+0,09$ & $-0,23$ \\
$4 \mathrm{~s}, \mathrm{a}_{1 \mathrm{~g}}$ & $-0,03$ & $+3,62$ & $-0,14$ \\
$4 \mathrm{p} 0, \mathrm{a}_{2 \mathrm{u}}$ & $-0,00$ & & $-0,00$ \\
$4 \mathrm{p} \pm 1, \mathrm{e}_{1 \mathrm{u}}$ & $-\mathbf{0 , 0 5}$ & $+4,72$ & $-0,29$ \\
gesamt & $+\mathbf{1 , 5 8 5}$ & & $+0,326$ \\
& at. E. & & at. E. \\
\hline
\end{tabular}

Tab. 2. Sternheimer-Korrektur der Feldgradientenbeiträge in $\mathrm{FeCp}_{2}$.

* Dies liegt nahe bei $Q=0,4 \mathrm{~b}$, dem Wert, den D. v. Ehrenstein, H. Kopfermann u. S. Penselin (Z. Phys. 159, 230 [1960]) erhielten, die den $\left\langle r^{-3}\right\rangle_{3 d}$-Faktor des Feldgradien-
Eine Sternheimer-Korrektur ist jetzt noch an dem Co-Quadrupolmoment (Abschnitt VII von Teil II) anzubringen. Der Konfiguration Co I $3 \mathrm{~d}^{7} 4 \mathrm{~s}^{2} \mathrm{a}^{4} \mathrm{~F}$ zufolge ist hier auch die $3 \mathrm{~d}^{5}{ }^{6} \mathrm{~S}$-Unterschale als zum polarisierbaren Rumpf gehörig anzusehen. Die für die Rumpfpolarisation maßgebliche Konfiguration ist Co III $3 \mathrm{~d}^{7}$, so daß auf einer Kurve gleicher Ionisationsstufe von Fe III $\gamma_{3 d}=-0,219^{4}$ aus mit der Neigung der entsprechenden $\gamma_{\infty}$-Kurve (Abb. 1) extrapoliert und Co III $\gamma_{3 \mathrm{~d}}=-0,208$ angenommen wird. Dann ist einerseits als neuer Wert für das Quadrupolmoment von Co

$$
Q_{\mathrm{Co}}=\frac{0,343}{\left(1+\gamma_{3 \mathrm{~d}}\right)}=0,433 \mathrm{~b} \text { * }
$$

einzusetzen, andererseits als neuer experimenteller Feldgradient in $\left(\mathrm{CoCp}_{2}\right)^{+}$e $q=1,67$ at. E. zu schreiben. Ihm steht der korrigierte theoretische Wert $e q=1,06$ at. E. gegenüber. In $\mathrm{FeCp}_{2}$ ist weiter $e q=1,25$ at. E. (Abschnitt VII von Teil II) als experimenteller Feldgradient anzusehen, der mit dem neuen theoretischen Ergebnis e $q=0,33$ at. E. zu vergleichen ist. In $\left(\mathrm{CoCp}_{2}\right)^{+}$liegt der experimentelle Wert um den Faktor 1,6 höher, in $\mathrm{FeCp}_{2}$ dagegen um einen Faktor 3,8. Was den größeren Fehler im zweiten Fall betrifft, ist zunächst zu bedenken, daß

1. für $\mathrm{FeCp}_{2}$ die Wahl der LCAO-Koeffizienten unsicherer war (Abschnitt $\mathrm{V}$ von Teil II) und davon bereits alle unkorrigierten Ergebnisse in Tab. 2 beeinflußt sind,

2. alle Zweizentrenbeiträge nach Maßgabe der Näherungsergebnisse geschätzt sind (Abschnitt VI von Teil II), wobei die Mulliken-Näherung mit dem größten Gewicht einging. Sie schätzt die Zweizentrenintegrale stark nach der Größe des jeweiligen Überlappungsintegrals ab, läßt daher die $3 \mathrm{~d}, 2$ p-Terme spürbar größer ausfallen als in $\left(\mathrm{CoCp}_{2}\right)^{+}$und führt außerdem zu dem fragwürdigen Ergebnis $q^{4 \mathrm{p} 0, \mathrm{a}_{2 \mathrm{u}}} \approx 0$.

Was die Sternheimer-Korrekturen selbst betrifft, so bestehen gegen den Ansatz (2) folgende Bedenken:

1. Wie Freeman und $\mathrm{W}_{\text {atson }}{ }^{8}$ gezeigt haben, werden die Wellenfunktionen der Zentralatomvalenzelektronen, wie sie der "restricted HartreE-FocK“Formalismus (RHF) produziert, im „unrestricted Hartree-Fock"-Prozeß stark von explizit behandelten (nicht über eine Schale gemittelten, wie im RHF-

ten aus der Feinstrukturaufspaltung bestimmten. In deren Parameter geht eine gewisse Rumpfpolarisation implizit ein (s. jedoch Freeman u. Watson ${ }^{8}$ ). 
Fall) Austauscheffekten beeinflußt, wodurch von vornherein die $\left\langle r^{-3}\right\rangle_{n l}$-Werte geändert werden. Sehr wichtig wird dann auch der Austausch mit den Rumpfelektronen, die $m$ und $m_{\mathrm{s}}$ mit Valenzelektronen gemeinsam haben. Durch diesen Austauscheffekt kann $\gamma_{n l}$ geändert werden, obwohl, wahrscheinlich aber zufällig, in Fe III $3 d^{6}$ die Störungsrechnung ${ }^{4}$ für eine Rumpfschale im Mittel das richtige Ergebnis trifft. Gerade $\gamma_{4 p}$ erscheint hier besonders beeinflußbar. Durch Austauschphänomene wird damit überhaupt die Verwendung einer nur ortsabhängigen, die Gesamtpolarisation des Rumpfes darstellenden Funktion $\gamma(r)$ in Frage gestellt.

2. Es wird angenommen, daß jede Ladung unabhängig von anderen vorhandenen den Rumpf stört. Eine solche einfache Superposition der Polarisationseffekte zerstört sicher die Selbstkonsistenz der gesamten Elektronenverteilung. Das Problem ist das gleiche wie z. B. für Fe IV $3 \mathrm{~d}^{5}$ in einem Kristall. Dort wird der Feldgradient am Fe-Kern zunächst allein von Gitterionen erzeugt, durch eine Gittersumme berechnet und dann einfach mit $\gamma_{\infty}$ korrigiert ${ }^{12}$. Auf diese Unsicherheit wird u. a. die Diskrepanz zwischen $Q$-Werten, die sich aus Fe IV- und Fe III-Ergebnissen ableiten, zurückgeführt.

Auffallend ist, daß gerade das Mitwirken rein äußerer Ladungsverteilungen bei der Erzeugung des Feldgradienten in Fe IV zu einem höheren Ergebnis für das Fe-Quadrupolmoment führt $\left(0,28^{13}\right.$ bis $0,4^{12}$ b) als im Fall von Fe III, wo man sich ihn nur vom sechsten 3 d-Elektron erzeugt denkt ${ }^{4}$. Ein solches höheres Quadrupolmoment würde den experimentellen Wert des Feldgradienten in $\mathrm{FeCp}_{2}$ verkleinern und damit den Unterschied zum theoretischen Ergebnis verringern.

Schließlich würde die Berücksichtigung der oben erwähnten Austauscheffekte in Co III $3 \mathrm{~d}^{7}$ die Radialfunktionen des 6-ten und 7-ten 3d-Elektrons gegenüber denen der $3 \mathrm{~d}^{5}$-Unterschale und der RHF-Funktion expandieren (vgl. die Cl-Ergebnisse von FrEEMan und $\mathbb{W}_{\text {ATson }}{ }^{8}$ ). Dadurch würde $\left\langle r^{-3}\right\rangle_{3 d}$ verkleinert, $Q_{\mathrm{Co}}$ weiter vergrößert und der experimentelle Feldgradient in $\left(\mathrm{CoCp}_{2}\right)^{+}$weiter verringert, womit auch hier eine bessere Übereinstimmung mit dem theoretischen Ergebnis erreicht würde.

Bisher nicht untersucht ist auch die Quadrupolpolarisation einer Valenzschale durch Elektronen in den übrigen Valenzschalen desselben Atoms und

12 G. Burns, Pliys. Rev. 124, 524 [1961]. durch die Überlappungs- und Liganden-Ladungsverteilung. Die diesbezügliche Aufgabe wäre, einen Quadrupolterm in den Hamilton-Operator für das Molekül aufzunehmen, ähnlich wie z. B. WAtson und Freeman $^{7}$ das für einen Atomrumpf und eine äußere Punktladung getan haben, und die üblichen Restriktionen des LCAO-Verfahrens aufzuheben.

\section{Schlußbetrachtung}

Die theoretische Beschreibung der Elektronenstruktur der Moleküle $\mathrm{FeCp}_{2}$ und $\left(\mathrm{CoCp}_{2}\right)^{+}$in den vorhergehenden Abschnitten der hier wiedergegebenen Arbeit ${ }^{10}$ ist besonders in folgenden Punkten kritisch zu beurteilen:

Die Bewegungsgleichungen der Elektronen sind nicht-relativistisch. Relativistische Effekte nehmen jedoch mit der Eintauchtiefe der Valenzelektronenwellenfunktionen zu (Abschnitt VI von Teil I).

Die Elektronen des beschriebenen atomaren oder molekularen Systems sind nur teilweise korreliert (Abschnitt VIII von Teil II).

Das Elektronenmodell wird als selbstkonsistent angesehen, wenn im Rahmen eines Variationsprozesses die Gesamtenergie des Systems minimalisiert ist. Dieses Kriterium ist für bestmögliche Elektronenwellenfunktionen relativ unscharf.

Die Wellenfunktionen der Elektronen werden als Linearkombinationen von Basis-Orbitals dargestellt. Dadurch wird die Variationsfreiheit eingeschränkt und in der LCAO-Näherung für Moleküle allgemein auf ein Valenzelektronenproblem reduziert. Die Mitvariation der Rumpfelektronenfunktionen ist an einfachen Molekülen durchgeführt worden.

Die Wahl einer der Molekülsituation angepaßten AO-Basis (Abschnitt III von Teil II) hat bei der Untersuchung der Überlappungsintegrale (Abschnitt IV von Teil II) beachtliche Verschiebungen aufgezeigt und damit die Bildung einer Basis aus AO's der Grundzustände freier Atome kritisiert. Von weiteren Anpassungsmöglichkeiten erscheint die Aufhebung der radialen Entartung von (nlm)-AO's in verschiedenen MO's am wirksamsten. Die Aufhebung dieser Entartung in den Rumpfschalen ermöglicht teilweise die selbstkonsistente Behandlung wichtiger Quadrupolpolarisationseffekte. Damit wird aber das Zentralfeldmodell und schließlich die Schalenstruktur des betrachteten Atoms zerstört, so daß charakteristi-

13 R. M. Sternheimer, Phys. Rev. 130, 1423 [1963]. 
sche Größen des freien Atoms nicht mehr auf seine Situation im Molekül übertragbar sind.

Die Quadrupolwechselwirkung wird aus der Gültigkeit der Laplace-Gleichung für das Volumen des betrachteten Kerns abgeleitet. Die endliche, gerade am Kernort selbst nicht verschwindende Ligandenelektronendichte in diesem Gebiet (Abb. 4 von Teil II) bedeutet, daß nicht mit dem ganzen Quadrupolglied der Entwicklung der elektrostatischen Wechselwirkung gerechnet wird.

Im LCAO-MO-Modell der Elektronenstruktur läßt sich der Feldgradient in charakteristische Beiträge entwickeln (Abschnitt VI von Teil II). Im Valenzelektronenteil ist der Feldgradient eines 4p-Elektrons nicht, wie früher angenommen wurde, vernachlässigbar, sondern von gleicher Größenordnung wie der eines 3d-Elektrons. In den betrachteten Molekülen verkleinern Zweizentrenbeiträge, die in der TownesDAILey-Theorie vernachlässigt werden, den resultierenden Feldgradienten um mindestens $30 \%$ gegenüber dem Valenzelektronenteil. Wegen der relativen Bedeutung der einzelnen Beiträge ist die genauest mögliche Aufschlüsselung (Abschnitte II und V von Teil II) der im LCAO-Modell implizit enthaltenen Elektronenverteilung erforderlich. Allein dadurch konnte die für $\left(\mathrm{CoCp}_{2}\right)^{+}$auffallende Nähe zum experimentellen Ergebnis erreicht werden.

Durch die im letzten Teil geschätzte Quadrupolstörung des Zentralatomrumpfes werden für $\left(\mathrm{CoCp}_{2}\right)^{+}$ experimenteller und theoretischer Feldgradient in gleicher Richtung und gleicher Größenordnung korrigiert. Diese Störung vergrößert im einzelnen die Zweizentrenbeiträge zum Feldgradienten erheblich, da die entsprechenden Ladungsverteilungen wesentlich außerhalb des Rumpfes liegen (Abb. 5 von Teil II) und dadurch mit starkem antishielding, d. h. mit einem effektiven Feldgradienten am Kernort erscheinen, der 5 -fach verstärkt gegen denjenigen ohne zwischengeschalteten Rumpf sein kann. Die Zweizentrenbeiträge inüssen deshalb genau berechnet sein; die Abschätzungen für $\mathrm{FeCp}_{2}$ reichen nicht aus.
Vorzeichen und Größe der Einzelbeiträge zum Feldgradienten deuten an, daß sich infolge der Molekülsymmetrie im Endergebnis Terme ähnlicher Größenordnung egalisieren können. Speziell das Beispiel des 4p-Valenzelektronenbeitrags zeigt, wie diese Tatsache eine so wünschenswerte Beurteilung wie die der hohen 4p-Besetzung im LCAO-Modell unmöglich macht.

Grundsätzlich reagiert der Feldgradient recht empfindlich auf die AO-Basis und den Aufbau der MO's aus dieser Basis. Wie sich Unsicherheiten in letzterer Hinsicht auswirken, zeigt der für $\mathrm{FeCp}_{2}$ auffallend schlechtere theoretische Feldgradient als der für $\left(\mathrm{CoCp}_{2}\right)^{+}$. Im einzelnen dürfte die Empfindlichkeit des Feldgradienten mit abnehmender Molekülsymmetrie immer deutlicher werden.

Dem Direktor des Physikalisch-Chemischen Instituts, Herrn Prof. Dr. G.-M. Schwab, danken wir für die Ermöglichung dieser Arbeit und das Interesse, das er ihr entgegenbrachte. Vorabdrucke von Veröffentlichungen übersandte uns freundlicherweise Herr Dr. R. E. Watson.

A n m. b. d. Ko r r. : C. J. Ballhausen und R. D. Fischer erhielten folgende neuen Ergebnisse (private Mitteilung):

1. Für Überlappungsintegrale in $\mathrm{FeCp}_{2}$, die denen der entsprechenden Rechnung von Shustorovich und Dyatkina (l. c. in Teil II) nahezu gleich sind, liefert die Wolfsberg-HenmноLz-Näherung, die annimmt, daß alle Wechselwirkungsintegrale proportional zu den Überlappungsintegralen sind (Abschnitt IV von Teil II), Energien der besetzten MO's, die mit den von Shustorovich und Dyatkina nach dem SCF-LCAOMO-Verfahren berechneten gut übereinstimmen. Dies bedeutet eine gewisse Stütze für die Annahme in Abschnitt IV von Teil II, aus der Nachbarschaft unserer Überlappungsintegrale für $\left(\mathrm{CoCp}_{2}\right)^{+} \mathrm{zu}$ denen von DaHL und Ballhausen (l. c. in Teil II) für $\mathrm{FeCp}_{2}$ auf eine Gleichheit der MO-Energien und mit einer gewissen Unschärfe auch der LCAO-Koeffizienten der MO's selber zu schließen.

2. Die Überlappungsintegrale (und die Wechselwirkungsintegrale) von D $\mathrm{DHL}_{\mathrm{H}}$ und Ballhausen enthalten keine Ringkorrektur (Dunitz und ORgel, l. c. in Teil II). Dadurch wird der physikalische Wert ihrer Ergebnisse eingeschränkt. Mathematisch sind diese Integrale von $D_{A H L}$ und Ballhausen für uns günstig, indem wir so gemäß der WolfsBerg-HelmholzNäherung die errechneten $\mathrm{FeCp}_{2}$-Ergebnisse auf $\left(\mathrm{CoCp}_{2}\right)^{+}$ übertragen konnten. Die Interpretation von $\mathrm{FeCp}_{2}$ ist aber mangelhaft, was auch unsere Ergebnisse andeuten.

Abschließend sei noch auf die Auswirkungen eines erweiterten AO-Basissatzes auf den Feldgradienten in $\mathrm{LiH}$ und eine Kritik der Sternheimer-Korrektur hingewiesen (S. L. KAhalas u. R. K. Nesbet, J. Chem. Phys. 39, 529 [1963]). 